\author{
RICYDE. Revista Internacional de Ciencias del Deporte \\ doi: $10.5232 /$ ricyde \\ Rev. Int. cienc. deporte
}

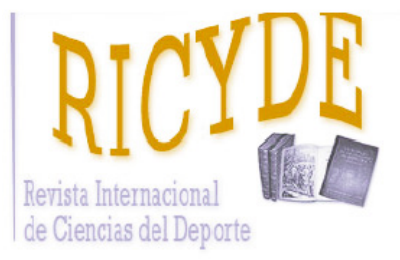

RICYDE. Revista Internacional de Ciencias del Deporte Volumen XIV - Año XIV

Páginas: $344-357$ - ISSN: $1885-3137$

Número 54 - octubre 2018

\title{
Historia y circunstancias sociales, deportivas y económicas del boxeo amateur español entre 1960 y 1980 \\ History and social, sports and economic circumstances of the Spanish amateur boxing between 1960 and 1980
}

\author{
María Teresa Calle-Molina, María Eugenia Martínez-Gorroño \\ Universidad Autónoma de Madrid. España
}

\begin{abstract}
Resumen
A través de esta investigación histórica se han conocido las circunstancias sociales, deportivas y económicas del boxeo amateur y olímpico español de los años sesenta y setenta del pasado siglo. El testimonio recabado a través de entrevistas de diez boxeadores españoles del periodo, siete de ellos olímpicos en una o varias ediciones de los Juegos, y los documentos recogidos de sus archivos personales, han sido contrastados con la información de la historia social y económica del periodo. El boxeo supuso una oportunidad formativa para jóvenes de sectores desfavorecidos en riesgo de vulnerabilidad social, con escasas oportunidades académicas y una incorporación laboral prematura. A pesar de hallar serias carencias en los recursos materiales y personales en el boxeo de aquel periodo, la orientación formativa del boxeo por parte de sus entrenadores aficionados transfirió valores y oportunidades que supusieron para aquellos jóvenes una orientación vital positiva y una promoción en su estatus social que, desde sus circunstancias familiares y la situación de la España del periodo, no hubieran conseguido de otra manera. Aquellas circunstancias, como resultado, ofrecieron hitos al boxeo olímpico español y unas oportunidades y experiencias para los boxeadores definitivas en sus trayectorias laborales y personales posteriores.
\end{abstract}

Palabras clave: boxeo amateur; boxeo olímpico; historia del deporte español; años sesenta; años setenta.

\section{Abstract}

Through this historical investigation, the social, sporting and economic circumstances of the amateur and Olympic Spanish boxing of the sixties and seventies of the last century have been studied. The testimony obtained through interviews of ten Spanish boxers from this period, seven of them Olympic in one or several editions of the Games, and the documents collected from their personal files, have been contrasted with the information of the social and economic history of the period. Boxing was an opportunity for young people from disadvantaged sectors at risk of social vulnerability, with scarce academic opportunities and premature employment to shape themselves. Despite finding serious shortcomings in the material and personal resources in boxing from that period, the pedagogical orientation of boxing by its amateur trainers transferred values and opportunities that meant these young people had a positive vital orientation and an advancement in their social status. They would not have achieved this otherwise because of their family circumstances and the situation of Spain in that period. Those circumstances, as a result, offered a milestone to the Spanish Olympic boxing and important opportunities and experiences for the boxers in their later work and personal path.

Key words: amateur boxing; Olympic boxing; history of Spanish sport; sixties, seventies.

Correspondencia/correspondence: María Teresa Calle-Molina

Universidad Autónoma de Madrid. España

Email: mariat.calle@uam.es 
Calle-Molina, M. T. y Martínez-Gorroño, M. E. (2018). Historia y circunstancias sociales, deportivas y económicas del boxeo amateur español entre 1960 y 1980. RICYDE. Revista internacional de ciencias del deporte. 54(14), 344-357. https://doi.org/10.5232/ricyde2018.05405

\section{Introducción}

A excepción de trabajos puntuales sobre los éxitos deportivos de ciertos boxeadores amateurs entre las décadas de los años sesenta y setenta que recogen brevemente los escasos triunfos deportivos (Antuña-Suárez, s.f.; Barbero, 1989; Tamayo-Fajardo, 2005; Díaz, 2008), no existe ninguna investigación acerca de la reconstrucción histórica de esta disciplina deportiva ni que la sitúe en el contexto social y deportivo en que se desarrolló. Con el objetivo de cubrir las lagunas existentes en el estudio histórico del boxeo español, presentamos una investigación inédita que recoge las circunstancias de diez boxeadores, siete de ellos deportistas olímpicos, que tuvieron su desarrollo como púgiles entre las décadas de los sesenta y setenta, así como los aspectos deportivos, sociales, económicos y culturales en los que se desenvolvieron y el papel educativo que el boxeo desempeñó en sus trayectorias vitales.

\section{Método de la investigación}

Metodológicamente este trabajo puede ser calificado como Historia del Tiempo Presente (HTP), ya que trata de comprender de manera global, articulada y formal una época histórica en la que vivieron sujetos que siguen vivos (Aróstegui-Sánchez, 2004). La coetaneidad de las fuentes orales en el mismo periodo objeto de estudio y su pertenencia temporal en la actualidad con el investigador (Soto-Gamboa, 2004) pone de relieve la importancia y la pertinencia de la proyección pública y académica de aquello que en la actualidad permanece poco estudiado $\mathrm{u}$ oculto (Bédarida, 1998). La HTP ha alcanzado un gran impulso internacional en las últimas décadas ya que este tipo de investigación brinda una oportunidad única para poder investigar con las fuentes orales protagonistas del periodo objeto de estudio y de recoger la memoria viva ya que generalmente, de no ser así, sus testimonios se perderían y con ellos una parte de la historia del deporte español.

Las técnicas de investigación utilizadas han sido el análisis documental de fuentes hemerográficas y bibliográficas, así como de otros documentos procedentes del archivo personal de los entrevistados. Por otro lado, a través de la entrevista semiestructurada de final abierto (Hammer y Wildavsky, 1990), se ha recogido el mayor grueso de la información, que por tanto, proviene de las fuentes orales protagonistas de los hechos históricos objeto de estudio. La información obtenida a través de los testimonios ha sido analizada y sometida a los procesos de crítica interna y externa (Best, 1982) para comprobar y garantizar la veracidad de las fuentes $\mathrm{y}$ de los contenidos de los relatos. La información procedente de las fuentes orales y documentales de diversa tipología ha sido contrastada y triangulada para conocer cuáles fueron los hechos personales y circunstanciales del objeto de estudio con el propósito de reconstruir de manera parcial aquel periodo dentro de los aspectos generales de la España de aquel momento.

Las fuentes orales fueron seleccionadas y categorizadas a partir de la característica común del inicio de su trayectoria en el boxeo amateur en España entre las décadas de los años sesenta y setenta, siendo por tanto fuentes primarias de los hechos históricos objeto de estudio. La configuración del guion de la entrevista se llevó a cabo teniendo en cuenta categorías estables y variables en función de la diferente tipología en la que se realizó la categorización de las fuentes a partir de los diferentes cauces que fueron tomando sus trayectorias deportivas. Así fue necesaria una categorización de las fuentes orales según su tipología, como se puede observar en la Tabla 1; siete de las diez fuentes orales pertenecieron a la Selección Nacional en el periodo objeto de estudio (teniendo en cuenta que los boxeadores olímpicos pertenecieron a la Selección Nacional), siete de ellas asistieron a unos JJOO y dos de ellas tomaron parte en dos ediciones consecutivas, siendo una de estas fuentes, Enrique Rodríguez Cal, primer medallista del boxeo olímpico español, con su medalla de bronce en Múnich-1972. Es necesario tener en cuenta que 
Calle-Molina, M. T. y Martínez-Gorroño, M. E. (2018). Historia y circunstancias sociales, deportivas y económicas del boxeo amateur español entre 1960 y 1980. RICYDE. Revista internacional de ciencias del deporte. 54(14), 344-357. https://doi.org/10.5232/ricyde2018.05405

fueron veintiséis los boxeadores olímpicos de aquellas dos décadas. Por último, tres de las fuentes fueron boxeadores aficionados. Los dos primeros consiguieron títulos a nivel estatal sin pertenecer a la Selección Nacional.

Tabla 1. Categorización de las fuentes orales según su tipología

\begin{tabular}{cl}
\hline & Categorización de las fuentes orales \\
\hline $\begin{array}{c}\text { Categoría 1 } \\
\text { Boxeadores olímpicos en dos } \\
\text { ediciones entre 1960 y 1980 }\end{array}$ & Enrique Rodríguez Cal y Antonio Rubio Fernández \\
\hline $\begin{array}{c}\text { Categoría 2 } \\
\text { Boxeadores olímpicos en una } \\
\text { edición entre 1960 y 1980 }\end{array}$ & $\begin{array}{l}\text { José Manuel Durán Pérez, Alfonso Fernández Fernández, Antonio Marcos } \\
\text { Chinea Hernández, Miguel Velázquez Torres y Moisés Fajardo Pérez }\end{array}$ \\
\hline $\begin{array}{c}\text { Categoría 3 } \\
\text { Boxeadores amateurs entre } \\
1960 \text { y 1980 }\end{array}$ & $\begin{array}{l}\text { José Ramón Gómez Fouz, Gonzalo Cortina Cueli y Antonio Domínguez } \\
\text { Gutrez }\end{array}$ \\
\hline
\end{tabular}

Siguiendo las directrices establecidas por la ética de la investigación histórica, las fuentes orales firmaron dos documentos previos a la entrevista en los que constaba el objetivo de reconstrucción histórica que pretendía la investigación y los permisos para realizar la grabación de su testimonio, así como para la consulta y utilización de su archivo personal. Se hizo constar la opción de poder cambiar y eliminar de manera parcial o total su aportación, aceptando que sus nombres y testimonios no fueran anónimos. Este último aspecto tenía el objetivo de darles voz y reconocimiento, en nuestra intención de llevar a cabo una recuperación histórica en concordancia con los mismos propósitos que en su momento Thompson (1988) confería al uso de la fuente oral en la investigación histórica, pues "le da vida a la propia historia ampliando así su enfoque" ya que "habla de personajes no sólo extraídos de entre los líderes, sino también de entre la mayoría desconocida de la gente" (Thompson, 1988, 36)

\section{Circunstancias sociales, económicas y culturales de los boxeadores: los entornos sociales y familiares}

Durante la década de los años 60 y hasta mediados de los años 70 del siglo XX, España permaneció bajo el régimen de la Dictadura del General Franco que se prolongaba aún desde 1939. En las décadas de los 50 y los 60 España se había desenvuelto en unas circunstancias de una seria precariedad económica, consecuencia en parte del aislamiento internacional y de los bloqueos económicos provocados por las respuestas ante el régimen político establecido en España. Sin embargo, con el avance temporal ya en la década de los 50, si bien la dictadura seguía impuesta como régimen de gobierno, el panorama empezó a mostrar algunos leves avances por las necesidades que el entorno de la economía occidental fue estableciendo. Principalmente "dos hechos marcaron la aceptación del Régimen en el mundo occidental: el Concordato con la Santa Sede y los Acuerdos con los Estados Unidos" (Castelló-Traver, 1988, 29) Así se permitió que España se integrara de forma relativa en los órganos internacionales a través de su adscripción al bloque geopolítico occidental y capitalista. 
Calle-Molina, M. T. y Martínez-Gorroño, M. E. (2018). Historia y circunstancias sociales, deportivas y económicas del boxeo amateur español entre 1960 y 1980. RICYDE. Revista internacional de ciencias del deporte. 54(14), 344-357. https://doi.org/10.5232/ricyde2018.05405

En las décadas objeto de estudio de este trabajo, la situación en España había experimentado cierta evolución como consecuencia, entre otros aspectos, de los efectos de los denominados "Planes de Desarrollo". En enero de 1964 se había iniciado el I Plan de Desarrollo Económico y Social, que tuvo continuidad con el II y el III Plan de Desarrollo aprobados en 1969 y 1972, respectivamente. Algunos autores especialistas en los procesos económicos del periodo afirman que en los años 70 se produjeron importantes cambios socioeconómicos en el país, "el proceso de desagrarización modifica la distribución de la población en España, al venir acompañado de movimientos migratorios interiores que implican elevados costes" (Biescas y Tuñón, 1980, 72), haciendo referencia a los importantes trastornos y sacrificios que supusieron para importantes grupos de población, desplazados de sus entornos habituales y la problemática sociocultural que implicaba la desubicación.

Ejemplo de aquellos datos aportados por la historiografía general, Enrique Rodríguez Cal, boxeador que ganó una medalla de bronce en los JJOO de Múnich de 1972 y participó en los de Montreal en 1976, al recordar la ubicación y el entorno en el que discurrió su infancia, hacía referencia a aquel proceso de migración interior, así como a la situación de precariedad económica y bajos recursos en los que se desenvolvió su familia. Su infancia transcurrió en Candás, a doscientos metros de la plaza de La Baragaña, donde nació. Cuando contaba cinco años su familia decidió mudarse a Valliniello, un sitio que él recordaba como "un poblado al lado del monte, de casas muy humildes, sin agua corriente" (Rodríguez-Cal en Cuervo, 2015a, 26). Allí vivían en dos habitaciones: una para los padres y otra para los siete hermanos, compartiendo además cocina con otra familia. Poco después, comenzaron a vivir en una de las casas del Barrio de la Luz en la ciudad de Avilés, producto del nuevo trabajo de su padre, ya que su empresa construyó hogares para los trabajadores (Rodríguez-Cal en Calle-Molina y Martínez-Gorroño, en prensa).

En el caso del boxeador Gonzalo Cortina Cueli, la situación fue muy similar. Este púgil recordaba sus orígenes humildes, tanto en su lugar de origen como en la ciudad a la que se trasladaron:

Nosotros descendemos, tanto por parte de familia de padre como de madre, del Busto en Villaviciosa, donde yo nací. Vengo de familia de agricultores, de toda la vida del campo. Hasta que mi padre y mi madre se trasladan a Gijón en el año sesenta y siete (G. Cortina-Cueli, entrevista personal, 26 de noviembre de 2016)

Antonio Rubio Fernández, boxeador que asistió a los JJOO en Múnich en 1972 y Montreal en 1976 fue también uno de aquellos niños parte del proceso migratorio interior. Cuando él tenía cuatro años de edad su familia, natural de Murcia, decidió mudarse a Mataró por cuestiones laborales y económicas.

Como síntesis de los resultados obtenidos, siete de los diez boxeadores entrevistados vivieron una o varias situaciones de movilidad. En todos los casos, el cambio de ubicación era motivado por la precariedad salarial o la falta de empleabilidad en sus lugares de origen. En uno de aquellos siete casos, el de Antonio Domínguez, su familia se trasladó a Alemania cuando éste era adolescente. Este último aspecto nos mostraba, dentro del colectivo estudiado, otro hecho histórico resaltado por los estudios historiográficos que evidencian que, en los años setenta empezaba a ser patente el inicio de la superación del aislamiento internacional, unido a que aún la mayor parte de la población se desenvolvía en unas circunstancias de serias carencias económicas, por lo que aproximadamente un millón de trabajadores españoles debieron salir a Europa contribuyendo con su sacrificio al llamado "milagro español” (Biescas y Tuñón, 1980, 72). 


\section{Trayectorias escolares y precocidad laboral de los boxeadores}

Profundizando en el conocimiento del nivel social, económico y cultural de los entornos familiares de aquellos deportistas, éstos ofrecen como resultado el perfil de unos jóvenes que mostraban dificultades de adaptación al entorno social de la nueva ubicación. Ello generaba el abandono del sistema educativo precozmente por falta de motivación y de conciliación social. Las diez fuentes orales entrevistadas, tuvieron una corta trayectoria escolar y se vieron impulsados a una inserción laboral prematura en actividades que no requerían cualificación y en las que percibían ínfimos ingresos, que sin embargo, resultaban definitivos en una economía familiar de recursos extremadamente humildes.

Rodríguez Cal, con catorce años dejó de asistir a la escuela para comenzar a trabajar como panadero. La partida de su hermano Avelino al Servicio Militar obligatorio implicó que la familia dejara de percibir su salario y por ello, fue imprescindible buscar remedio. A la una de la madrugada comenzaba su jornada hasta poco después de amanecer, haciendo vida normal hasta la tarde, cuando después de recorrer a pie unos cuatro kilómetros hasta Valliniello, comenzaba su entrenamiento deportivo. A los 16 años dejó la panadería para comenzar a trabajar en la construcción y en la Bilbaína de Montajes, una empresa subcontratada de una gran siderurgia. Con aquel cambio buscaba algo más de tiempo diario para dedicarle al boxeo (E. Rodríguez-Cal, entrevista personal, 4 de abril de 2015)

Antonio Marcos Chinea Hernández, boxeador que participó en los JJOO de México en 1968, recordaba aquellos momentos como: "complicados, difíciles,... prácticamente la posguerra, y había que trabajar todos los hermanos y todas las hermanas", ya que había una carencia importante de recursos básicos en su hogar que recordaba de la siguiente manera:

La comida nunca nos faltó, pero sí que faltó comprar unas alpargatas porque no teníamos para comprarlas. Y si faltaba para ir al cine, porque no teníamos para ir al cine. No podíamos ir al colegio. Entonces teníamos que arreglárnosla con lo que había, hacer intercambio de cosas que teníamos en los cultivos. Nosotros dábamos la cebolla y el ajo, y ellos nos daban los cereales para hacer el gofio ${ }^{1}$ para poder comer, o sea, la vida aquí era así (A.M. Chinea-Hernández, entrevista personal, 14 de enero de 2017).

Éste reconocía que aquella falta de recursos fue un aliciente definitivo para su incorporación al boxeo. En él veía una posibilidad laboral a largo plazo que le permitiría salir de aquel entorno que presentaba tantas carencias.

Debido a las carencias económicas, la escala de preferencias a la hora de elegir sus ocupaciones siempre partía de la laboral, teniendo que elegir el resto de las horas del día, entre la asistencia a la escuela o al gimnasio. Miguel Velázquez Torres, boxeador que asistió a los JJOO de Tokio en 1964, así lo recordaba:

Cuando no tenía nada que hacer yo iba a la escuela. Recuerdo una vez que mi padre me fue a buscar a la escuela para ir a trabajar a un bar a lavar los vasos y tenía ocho o nueve años... Entonces hice el graduado escolar con 40 años (M. Velázquez-Torres, entrevista personal, 4 de febrero de 2017).

\section{Su iniciación deportiva y las circunstancias del boxeo aficionado durante el periodo}

La iniciación deportiva de aquellos jóvenes en el boxeo también estuvo condicionada por las circunstancias económicas, ya que el hecho de que su práctica no implicara ningún gasto, fue un aspecto decisivo para su inicio en los entrenamientos. En sus testimonios fue señalado

\footnotetext{
${ }^{1}$ Alimento tradicional canario compuesto de harina de maíz, trigo o cebada tostados. Ocasionalmente, mezclados con azúcar.
} 
Calle-Molina, M. T. y Martínez-Gorroño, M. E. (2018). Historia y circunstancias sociales, deportivas y económicas del boxeo amateur español entre 1960 y 1980. RICYDE. Revista internacional de ciencias del deporte. 54(14), 344-357. https://doi.org/10.5232/ricyde2018.05405

especialmente el hecho de que no se pagaban cuotas mensuales para asistir a los gimnasios, ni era necesaria la compra de ninguna indumentaria. En el caso de los preparadores se realizaba desde un planteamiento generoso y altruista, ya que no tenían salario. Ejercían como entrenadores solo por afición.

José Durán Pérez, boxeador español que asistió a los JJOO en México en 1968 explicaba así aquellos aspectos: "no se pagaba nada, era gratis, solamente doscientas cincuenta pesetas al año que era la mutualidad deportiva por si te lesionabas y te cubría el seguro, elegí ese deporte por eso" (J.M. Durán-Pérez, entrevista personal, 11 de noviembre de 2016)

Otros boxeadores confirmaban las mismas circunstancias: “¿Quién tenía dinero para pagarse un gimnasio o ir a clases de tenis al grupo cultural Covadonga? Muy poca gente. Entonces allí... con unas alpargatas, un pantalón corto y una camiseta tú ya estás entrenando boxeo" (G. Cortina-Cueli, entrevista personal, 26 de noviembre de 2016)

La iniciación deportiva de aquellos jóvenes en el boxeo con frecuencia fue consecuencia de la influencia de familiares o amigos y propiciada por la cercanía de uno de los incipientes gimnasios de boxeo a su ubicación familiar. Así Enrique Rodríguez Cal empezó llevando la ropa a su hermano mayor al gimnasio y así se introdujo en aquel deporte. Aquello le suponía caminar cuatro kilómetros, ya que la economía familiar no le permitía ningún gasto suplementario "no teníamos esas tres pesetas o dos, no recuerdo muy bien, que valía el autobús" (Rodríguez-Cal, entrevista en Televisión Asturias, 2008).

Así, empezó con 13 años a entrenar en un gimnasio ubicado en una antigua cárcel que se mantenía en condiciones muy precarias, el "Centro Deportivo", que pertenecía al Sindicato Vertical $^{2}$ (Del Río, 2012). Era un lugar al que se accedía por la entrada de un bar, conocido en Avilés como "El Hogar del Productor". Era un patio al aire libre, con el suelo de cemento, con poco más material que un saco que colgaba de un árbol. Los guantes eran los mismos para todos, de uso común y de talla única. El vestuario era el almacén del bar donde se apilaban las cajas, allí podían cambiarse y dejar su ropa colgada de un "puntal"3. No había duchas ni agua caliente (E. Rodríguez-Cal, entrevista personal, 4 de abril de 2015)

Las circunstancias de otros boxeadores del periodo eran muy similares. Antonio Domínguez Gutiérrez, que se inició como boxeador aficionado en los sótanos del Hogar de Ciares, ubicado entonces en la calle Ramón y Cajal de Gijón, (Asturias) recordaba:

De aquella no había ni para bañarse, o sea no había ni duchas. Llegabas, sudabas y después para casa, te bañabas en casa. Luego cuando cambiamos ya fuimos a un gimnasio que tenía por lo menos agua. Era un depósito de agua como en las casas de aldea. Era agua, un grifo y además agua fría. Era lo que había... (A. Domínguez Gutiérrez, entrevista personal, 9 de octubre de 2015).

Por su parte Marcos Chinea, recordaba los lugares donde se llevaban a cabo los combates en los que se iniciaban como boxeadores. En su caso, era un club de billar, en el cine Avenida, en el parque San Telmo del Puerto de la Cruz. También recordaba encuentros en las fiestas de los pueblos. Allí se improvisaban las sillas de los asistentes y un cuadrilátero muy rudimentario para que pudieran boxear.

\footnotetext{
${ }^{2}$ Conocida como la Organización Sindical Española, única organización sindical autorizada legalmente durante el periodo franquista español.

${ }^{3}$ Clavo largo insertado en la pared de ladrillo visto, descrito por Rodríguez Cal en la entrevista realizada el 4 de abril de 2015 .
} 
Calle-Molina, M. T. y Martínez-Gorroño, M. E. (2018). Historia y circunstancias sociales, deportivas y económicas del boxeo amateur español entre 1960 y 1980. RICYDE. Revista internacional de ciencias del deporte. 54(14), 344-357. https://doi.org/10.5232/ricyde2018.05405

La escasez de materiales y las carencias en general eran solventadas en múltiples ocasiones a partir del ingenio de ellos mismos. Chinea Hernández mencionaba el material que para uso común idearon como mancuernas, que construían con tubos y latas de leche condensada rellenos de hormigón.

Allí ponían el saco, la pera, los guantes y las guantillas, el resto teníamos nosotros que comprarlos con sacrificio, porque no había dinero para comprarlos. Yo llevaba unas alpargatas de esparto, porque no tenía otra cosa, yo no tenía para comprar (A.M. Chinea-Hernández, entrevista personal, 14 de enero de 2017).

En otros casos, como el de Moisés Fajardo Pérez, olímpico en los JJOO de Méjico en 1968, recordaba que incluso eran los propios boxeadores que se iniciaban los que montaban el gimnasio de manera progresiva, con donaciones de los vecinos de sus localidades y con grandes esfuerzos personales.

José Durán Pérez, en la entrevista que con él mantuvimos, reflexionaba que, si bien en la actualidad cada boxeador tiene su material individual, ese aspecto en aquel momento era impensable. Antonio Domínguez Gutiérrez, nos explicó que su indumentaria para ir a entrenar se la confeccionaron sus hermanas que le hicieron unos pantalones y una camiseta. Por aquella absoluta precariedad, Gonzalo Cortina recordaba que cuando lograban tener indumentaria de boxeo, quedaba guardada en el armario a la espera de un encuentro importante y no la usaban para entrenar. Aquellos testimonios han sido contrastados con numerosas fuentes hemerográficas que relataban las circunstancias del pugilismo en la España de aquel momento.

A pesar de aquellas circunstancias, el boxeo amateur consiguió grandes retos nacionales e internacionales logrando durante la década de los setenta "los años de mayor actividad y brillantez" (Sánchez García, 2009, 7) los cuales influyeron positivamente en la participación española en boxeo en los JJOO. Específicamente, se desarrollaron cinco sucesos definitivos en aquel periodo: la creación del Colegio Nacional de Preparadores de boxeo en 1968 (Alfil, 1968), la celebración de la Asamblea del Bureau Continental Europeo de boxeo en 1970 en el Instituto Nacional de Educación Física (INEF) en Madrid (ABC, 1970), la celebración de un campeonato de Europa de boxeo Amateur por primera vez en España en 1971 (RTVE, 1971), la creación de la Liga Nacional de Boxeo Aficionado en 1972 y, por último, la organización del primero curso nacional para preparadores de boxeo en 1972 (FEBOX-BOXEO, 1972)

\section{Los entrenamientos}

Las rutinas de entrenamiento eran recordadas como muy rudimentarias, repetitivas y muy similares, aspectos asociados con las características de los preparadores. Los datos recogidos en referencia a los entrenadores les definieron con un perfil de personas muy entusiastas que contaban con una formación totalmente autodidacta. Solían redundar siempre en los mismos ejercicios y gestos que habían visto hacer o habían vivido como antiguos boxeadores. Podemos afirmar que en el periodo objeto de estudio tampoco existían especialistas. Hemos podido documentar que para solventar aquella circunstancia del 7 al 19 de febrero de 1972, la Federación Española de Boxeo a través de la Escuela Nacional de Boxeo, llevó a cabo el primer curso de boxeo dirigido a preparadores y ayudantes, que tuvo lugar en el INEF de Madrid (Amorós, 1972)

Las diez fuentes orales tuvieron unas circunstancias muy similares en todos los casos, a pesar de provenir de diferentes lugares de España. Todos los entrevistados aludían a la carencia de instalaciones apropiadas para la práctica deportiva, la falta de recursos materiales en los gimnasios y la inexperiencia de sus preparadores. 
Calle-Molina, M. T. y Martínez-Gorroño, M. E. (2018). Historia y circunstancias sociales, deportivas y económicas del boxeo amateur español entre 1960 y 1980. RICYDE. Revista internacional de ciencias del deporte. 54(14), 344-357. https://doi.org/10.5232/ricyde2018.05405

También fue mencionado, como consecuencia de las precariedades económicas de los propios practicantes y sus entornos familiares, el hecho de que no podían tener una dieta específica. La compra de los productos alimenticios básicos era lo que se podían permitir las familias, y cualquier tipo de adquisición diferente, era considerado un lujo imposible. Antonio Domínguez Gutiérrez, así lo confirmaba: "éramos gente que trabajaba o para ayudar en casa o para mantenerse. La gente no podía comer lo que quisiera, de aquella ya bastante régimen tenías con lo que había en casa" (A. Domínguez-Gutiérrez, entrevista personal, 9 de octubre de 2015).

\section{Su evolución económica y social. La oportunidad que supuso el boxeo}

Durante aquel periodo se organizaban con frecuencia torneos pugilísticos. A partir de los datos que ofrecen las fuentes hemerográficas hemos podido documentar los que alcanzaron más popularidad: el Torneo Guante de Oro, organizado en Guipúzcoa; los Torneos Costa del Sol y Antonio González organizados en Almería; el Torneo Filomatic en Cataluña; el Torneo Roberto Duque en Alicante o el Torneo Paulino Uzcudun celebrado en Madrid. Estos eran organizados por las diversas federaciones regionales y enfrentaban a los gimnasios de localidades cercanas que podían o no pertenecer a la misma ciudad. En el caso del último torneo citado competían los gimnasios "Chamberí", "Atlético de Madrid", "La deportiva Ferroviaria", "Club Acude" y "Club Basterra". Se fue generando así una red de encuentros en la que los boxeadores amateur comenzaban a competir con púgiles de un entorno y nivel similar (FEBOX-BOXEO, 1970 a y b).

Además de aquellos torneos, organizados de manera sistemática, tenían lugar también veladas esporádicas. Éstas podían ser mixtas, es decir, con combates de boxeadores amateurs y profesionales o sólo de carácter aficionado. Los escenarios en los que se organizaban eran múltiples y de diversa naturaleza. Algunas se hacían en instalaciones deportivas del Frente de Juventudes ${ }^{4}$ (Montañés, 1970), en frontones, salas de baile, plazas de toros, piscinas al aire libre, circos, sótanos o plazas de los pueblos.

Los púgiles que lograban destacar en aquellos encuentros comenzaban a presentarse a los campeonatos interregionales en los que se decidía qué boxeador iría en cada uno de los pesos a los campeonatos de España dentro de cada una de las federaciones regionales (FEBOXBOXEO, 1970c). Los Campeonatos de España de Boxeo Aficionado, lo que suponía el mayor reto para un boxeador amateur en el inicio de su trayectoria pugilística, eran organizados anualmente por la Federación Española de Boxeo. El gran respaldo institucional que tuvieron puede apreciarse a partir de analizar ciertos aspectos como la conformación de su Comité de Honor que además de estar integrado por los diversos comités técnicos, estaba presidido por el General Francisco Franco, el ministro-secretario general del Movimiento ${ }^{5}$ y por el Delegado Nacional de Educación Física y Deportes. En aquellos Campeonatos de España se disputaban los títulos individuales por pesos. Potenciando su importancia en 1940, se creó la Copa de S.E. el Generalísimo con la que se premiaba a los equipos, compuestos por los boxeadores de una misma Federación Regional, que ganaran dos campeonatos de España consecutivos o tres alternos (Meyerd y Girard, 1966).

\footnotetext{
${ }^{4}$ Sección del partido político Falange Española Tradicionalista y de las Juntas de Ofensiva Nacional Sindicalista (JONS) creada por el régimen de Franco con objeto de adoctrinar políticamente a los jóvenes españoles conforme los principios del Movimiento Nacional.

${ }^{5}$ El Movimiento Nacional o «Movimiento» era el nombre que recibía durante el periodo dictatorial franquista el mecanismo totalitario constituido por los múltiples organismos, sindicatos, cargos públicos y el único partido político existente "Falange Española Tradicionalista y de las Juntas de Ofensiva Nacional Sindicalista" con objeto de ser el único cauce de participación en la vida pública española.
} 
Calle-Molina, M. T. y Martínez-Gorroño, M. E. (2018). Historia y circunstancias sociales, deportivas y económicas del boxeo amateur español entre 1960 y 1980. RICYDE. Revista internacional de ciencias del deporte. 54(14), 344-357. https://doi.org/10.5232/ricyde2018.05405

Para los boxeadores amateur se fueron propiciando unas experiencias y oportunidades que, a partir de sus testimonios sabemos que para ellos eran totalmente extraordinarias e impensables en las circunstancias en las que sus entornos familiares se desenvolvían. Se les ofrecieron oportunidades de viajar y conocer otros lugares del país, a veces visitando zonas muy cercanas, pero que suponían viajes totalmente extraordinarios entre todas las personas de sus ambientes cotidianos. Gran parte de ellos, incluso viajaron a países extranjeros, aspecto a priori ni siquiera imaginable en las circunstancias en las que se encontraba la mayor parte de los ciudadanos españoles.

Como ejemplo, Rodríguez Cal, gracias a su práctica deportiva pudo conocer otros lugares de España. Viajó a Galicia en los encuentros interregionales. Como consecuencia de sus triunfos, pudo viajar a la capital, viaje que pudo pagar por el dinero para el billete que le proporcionó la Federación Asturiana. Recordaba especialmente la gran ilusión de su primer viaje a Madrid. Fue alojado en el hotel San Antonio de la Florida. Le llevaron al sastre a hacerle un traje a medida: chaqueta azul, pantalón gris, camisa blanca y corbata con las rayas de la bandera española (Rodríguez-Cal en Cuervo, 2015a). Experiencias extraordinarias que reforzaban su autoestima, le abrían a otras realidades y eran un motivo de orgullo especial para él y sus familiares.

En 1970 como consecuencia de su participación en el I Campeonato de Boxeo de Europa Junior, para menores de veintiún años, celebrado en noviembre de 1970 en Miskolc, Hungría, hizo su primer viaje al extranjero. En una de las entrevistas que mantuvimos rememoraba cómo llevaba la chaqueta azul, con el escudo en el pecho, entrando en un aeropuerto y volando por primera vez acompañando al equipo nacional aún como suplente (Cuervo, 2015b). Posteriormente empezó a formar parte del equipo olímpico de manera intermitente, aspecto que le llevó a participar en encuentros que se disputaron en Francia o Marruecos (Barbero, 1989).

En las concentraciones los integrantes del Equipo Olímpico disfrutaban de estancias en hoteles y se desenvolvían en ambientes con unas circunstancias de vida y unas comodidades cotidianas que eran bien diferentes de las que vivían en sus hogares y en sus entornos de origen. Aquellos primeros viajes a otros lugares, fuera de sus núcleos familiares, comenzaron a llenar de experiencias a aquellos jóvenes que, una vez eran admitidos por la Selección Nacional, comenzaban a viajar a países extranjeros, aspecto que hubiera sido de otra manera totalmente impensable para ellos. José Durán recordaba que su primer viaje en avión fue con la selección al Campeonato de Europa de Boxeo Amateur celebrado en Roma, pidiendo un permiso especial para poder ausentarse de su Servicio Militar (J.M. Durán-Pérez, entrevista personal, 11 de noviembre de 2016)

De la misma manera, Marcos Chinea Hernández recordaba especialmente su primer viaje en avión a Atenas cuando le llamaron de la Selección Española en 1967, acompañados por el preparador Enrique Soria.

Fue mi primer viaje que hice al extranjero, te puedes imaginar: la Acrópolis de Grecia y ver Atenas desde arriba para abajo, para mí fue una pasada [...] Cuando llegué de Grecia no te puedo contar, porque los amigos me preguntaban cómo era eso, cómo era Grecia... El boxeo me dio la oportunidad de conocer a mucha gente (A.M. Chinea-Hernández, entrevista personal, 14 de enero de 2017).

Formar parte de aquella selección de boxeadores que comenzaban a viajar también supuso para ellos disfrutar de vestuario deportivo de calidad que en aquellos momentos era un lujo inaccesible para gran parte de los jóvenes españoles de su entorno. Una vez que pertenecían a la Selección Nacional y se concentraban para preparar algún campeonato importante, además, percibían una ayuda económica, que aunque no era cuantiosa, si era significativa en sus economías, y les permitía ayudar a sus familias. Chinea Hernández lo recordaba así: 
Nos daban la comida, nos daban los gastos de la comida allí y luego, ya con las quince mil pesetas que me daban, le mandaba siete mil quinientas pesetas a mi madre y siete mil me quedaba yo, y ahorré dinero... (A.M. Chinea-Hernández, entrevista personal, 14 de enero de 2017).

A pesar de aquellas ventajas, las condiciones de los entrenamientos y de los gimnasios que se disponían para la Selección Nacional, eran recordadas como condiciones muy similares a las de sus entornos de procedencia, pues aquella era la situación general en la que se desenvolvían los deportes en aquel periodo.

\section{El deporte como medio educativo y oportunidad vital}

La información recogida, en cuanto a la formación académica de los púgiles entrevistados fue que se iniciaron en el boxeo habiendo superado escasamente los estudios primarios. Su corta trayectoria escolar, unida a la nula cualificación profesional, les daba acceso únicamente a empleos cuyos salarios servían para completar las necesidades básicas familiares, que se cubrían con las aportaciones económicas de varios miembros de la familia. Para las diez fuentes orales de este estudio, el acceso al deporte fue una oportunidad única de recibir una educación en valores por parte de sus preparadores. Aquellos entrenaban por afición, pues no percibían remuneración alguna por su trabajo en los diez casos estudiados.

Recogemos como ejemplo el testimonio de Antonio Domínguez, quien reflexionaba en cuanto que el boxeo supuso una herramienta de superación y de toma de confianza que estaba necesitando, ya que en la escuela tuvo dificultades de adaptación. Además, recapacitaba sobre cómo aquel deporte le apartó de otras prácticas nada positivas, como beber alcohol o fumar:

El gimnasio me recuerda cosas buenas, primero creo que gracias al gimnasio no bebo, yo ni bebía ni fumaba y yo era feliz. Me valió, me defendía, y... nunca abusé. Eso eran normas que me dijo el entrenador "tu procura si te pegan esquivar, evitar. Tú procura no soltar la mano, nunca" (A. Domínguez-Gutiérrez, entrevista personal, 9 de octubre de 2015).

Marcos Chinea, desde su perspectiva y las características de su entorno recordaba como el boxeo le proporcionó motivación e ilusión, poder conocer gente, competir y tener una oportunidad de romper con las circunstancias económicas y la carencia formativa y académica en la que se encontraba:

El boxeo me hizo crear una disciplina que luego me valió para el resto de mi vida. [...] Yo enfoqué mi propia vida como si fuera un combate. Ha sido la disciplina, el respeto al compañero, a todo lo que te rodea, la constancia en la vida del deporte, la disciplina, el trabajo, ha sido para mí importante (A.M. Chinea-Hernández, entrevista personal, 14 de enero de 2017).

Gonzalo Cortina Cueli, en este mismo sentido reflexionaba que aquella práctica deportiva le proporcionó ciertas oportunidades formativas. Hoy en día consideraba que el boxeo fue un definitivo impulso en su trayectoria vital:

El boxeo me hizo conocer a tanta gente... y me abrió puertas. Sí, es que tengo que estar agradecido al boxeo. Conocí mucha gente, amigos, viví momentos entrañables, sigo vinculado al boxeo desinteresadamente (G. Cortina-Cueli, entrevista personal, 26 de noviembre de 2016).

Además de aquellos aprendizajes por parte de sus entrenadores, hemos podido conocer a través del archivo personal de Marcos Chinea Hernández la circunstancia de que a los miembros de la Selección Nacional de 1968 se les impartió un curso de electricidad costeado por la Federación Española de Boxeo (FEB). En la concentración realizada en Pontevedra, después de los entrenamientos aquellos jóvenes asistieron al curso desarrollado por la escuela sindical para obtener una certificación oficial de montador electricista y embobinador industrial como se puede observar en la Figura 1. De aquella manera los jóvenes boxeadores pudieron aprender un oficio que pudiera permitirles elevar su estatus profesional y desempeñar un puesto de trabajo mejor remunerado a su vuelta en sus lugares de origen (ABC, 1968). 


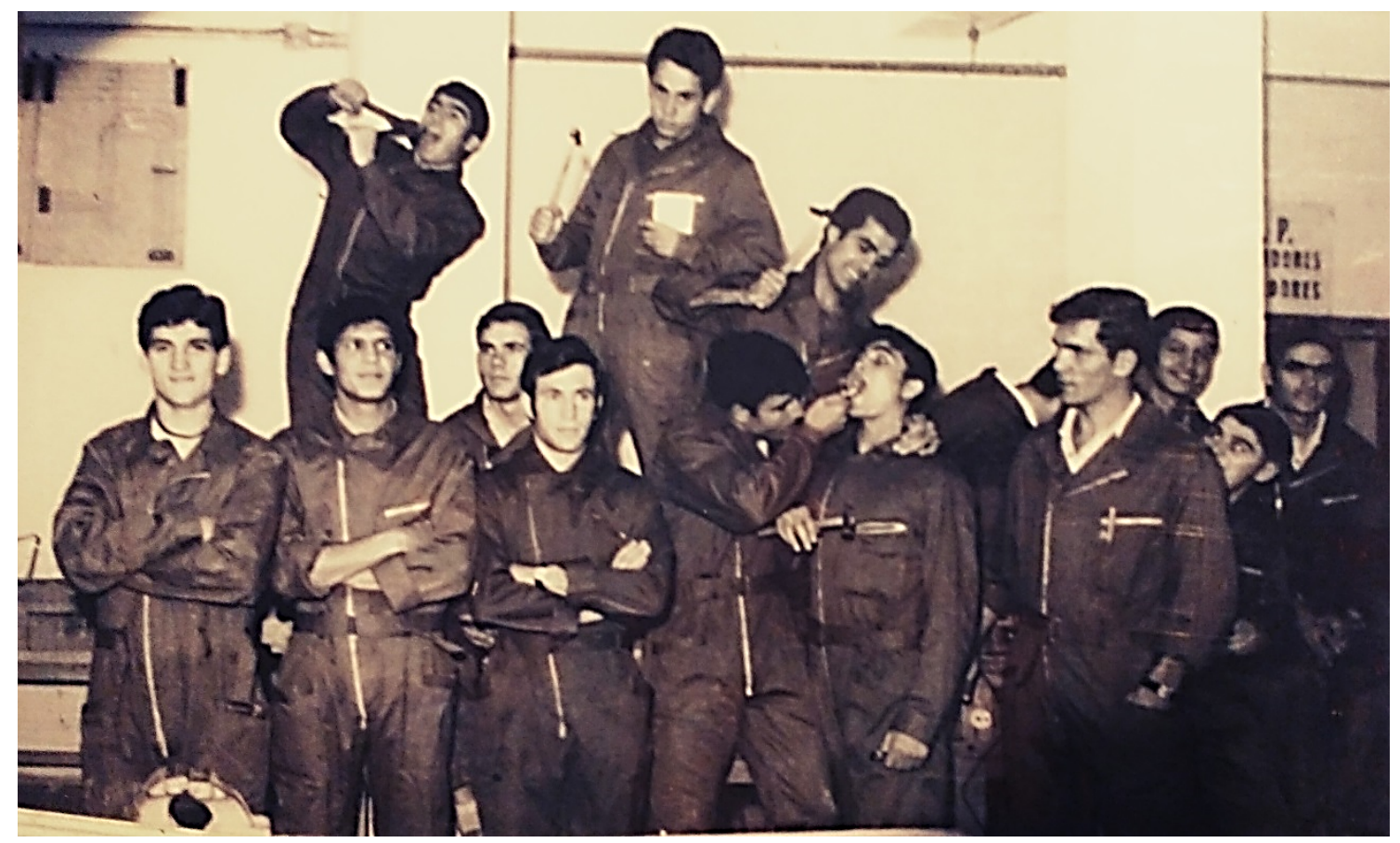

Figura 1. Boxeadores preseleccionados para asistir a los JJOO en México en 1968. Realizando un curso de electricidad costeado por la FEB en el momento de concentración. Imagen del archivo personal de Antonio Marcos Chinea Hernández.

El boxeo permitió a los diez boxeadores entrevistados, cuyas posibilidades económicas les permitían cubrir apenas las necesidades personales, realizar unas actividades que les sirvieron para formarse, aprender a desenvolverse y conocer otras realidades a las que nunca habrían tenido acceso a partir de su ubicación social. La práctica del boxeo fue una oportunidad de superación económica y cultural.

\section{Sus circunstancias finales y el impulso experimentado}

Los entrevistados que fueron boxeadores aficionados en las décadas de los sesenta y setenta en España, y que perseveraron hasta alcanzar algún éxito deportivo, generalmente obtuvieron una elevación del estatus social propiciada por las oportunidades de experiencias personales, así como de ingresos económicos. Así el boxeo fue la opción que orientó positivamente su trayectoria vital en los diez casos estudiados. Su tenacidad en la práctica del boxeo como aficionados fue definitiva para conseguir éxitos personales, sociales e incluso laborales. En los diez casos, estos boxeadores afirmaron que el boxeo supuso una plataforma positiva que orientó su futuro académico, laboral, personal y deportivo.

Marcos Chinea Hernández, tras su retirada del Equipo Olímpico después de los Juegos de Méjico en 1968, recordaba cómo el boxeo le proporcionó un cambio en su estatus social y, por tanto, una oportunidad laboral que de otra manera no se le hubiera ofrecido teniendo en cuenta su procedencia y formación académica:

A mí se me respetó mucho en la vida por haber sido olímpico. Yo iba a cualquier reunión, a cualquier sitio y decían "Marcos Chinea fue campeón de España y olímpico" Ya ahí me trataban y me miraban de otra forma. Después en mi vida particular y como persona me ayudó porque yo iba a buscar trabajo y me recomendaban porque, "mira Marcos Chinea fue olímpico, campeón de España..." (A.M. ChineaHernández, entrevista personal, 14 de enero de 2017).

De forma similar, Rodríguez Cal, el haber ganado la primera medalla de la historia del boxeo olímpico español, le supuso un impulso definitivo en su trayectoria posterior para poder buscar 
Calle-Molina, M. T. y Martínez-Gorroño, M. E. (2018). Historia y circunstancias sociales, deportivas y económicas del boxeo amateur español entre 1960 y 1980. RICYDE. Revista internacional de ciencias del deporte. 54(14), 344-357. https://doi.org/10.5232/ricyde2018.05405

un empleo y aprovechar el reconocimiento social de aquel momento. Además, le ayudó a percibir un dinero que, en su situación personal, le facilitó el poder costear aspectos vitales:

Cuando gané la medalla de bronce, yo fui con veinte años... Un chaval sin trabajo, recién casado, iba a ser padre... no tenía trabajo, me habían llamado para ir para la mili, o sea yo lo tenía muy negro. Y la medalla, me abrió la vida, me abrió las puertas... O sea que yo tengo que estar, pues muy agradecido, "me puso a vivir", (Rodríguez-Cal, 2017).

Aquellos triunfos en el boxeo amateur y el estatus que suponía asistir a unos Juegos Olímpicos, a otros boxeadores les ayudó a comenzar su carrera en el boxeo profesional, con lo que consiguieron elevar sus ingresos, convirtiéndose en su ocupación laboral, como fue el caso de José Durán. El boxeo profesional ofrecía otra situación y otras circunstancias distintas a las que han sido descritas en este trabajo.

\section{Discusión}

Las limitaciones de las que partía esta investigación eran las escasas fuentes documentales disponibles en los archivos institucionales, así como la dificultad en el acceso a las fuentes orales. Son limitados los boxeadores olímpicos de aquel periodo. La obligada "crítica externa" de la fuente que propone Best (1982) estaba condicionada por su elevada edad y por el acceso a su memoria, pues los hechos que pretendíamos recuperar sucedieron con cierta lejanía temporal. Para ello, simultáneamente a la realización de las entrevistas se utilizó la "estimulación del recuerdo" indicada a partir de la metodología propuesta por MartínezGorroño y Hernández-Álvarez, (1999) a través de la documentación gráfica que fuimos rescatando del archivo personal de las fuentes orales.

La recuperación de la información facilitada a través de los testimonios de las fuentes orales vivas, el rescate de la documentación depositada en sus archivos personales, suponen un conjunto de fuentes primarias definitivas, que de no haber sido recuperadas para este trabajo quedarían perdidas en tan solo unos años. Igualmente, con este trabajo de recuperación histórica pretendemos llevar a cabo un reconocimiento necesario y justo para aquellos deportistas que realizaron enormes esfuerzos personales en una etapa de nuestra historia que ofrecía múltiples dificultades que sin duda fueron superados y ofrecieron para nuestro país unos éxitos deportivos escasamente recordados y sobre los que no existen investigaciones en este momento. Para investigaciones futuras en esta línea recomendamos consultar las fuentes orales disponibles en las cuatro ediciones de los Juegos entre 1960 y 1976 a través de entrevistas para, de esta forma, intentar reconstruir un deporte poco estudiado que fue muy popular en la historia del deporte español en aquel periodo, así como rescatar sus archivos personales pues, de otra manera, se perderá una documentación de gran valor histórico.

\section{Conclusiones}

El boxeo amateur español, durante las primeras décadas de la etapa de la dictadura que sucedió a la Guerra Civil Española, que proporcionó 26 boxeadores olímpicos y la primera medalla española en unos Juegos se desenvolvió generalmente con jóvenes de escasa trayectoria académica, procedentes de familias económicamente muy desfavorecidas que se reubicaban en los entornos periféricos alrededor de ciertas ciudades, que acogían poblaciones inmigrantes atraídas por los puestos de trabajo que ofrecían las incipientes industrias.

Aquella frágil economía familiar impulsaba a los jóvenes a una inserción laboral precoz, con salarios ínfimos que les permitía pocas oportunidades de progreso. Debido a aquella falta de recursos, en los diez casos estudiados, propició la iniciación al boxeo amateur en los gimnasios cercanos a sus viviendas, pues no se cobraban cuotas mensuales y no se necesitaba material deportivo. 
Todos los testimonios recogidos recordaban sus entrenamientos en gimnasios mal acondicionados para la práctica deportiva, sin zonas de aseo preparadas, con grandes precariedades y escasos materiales deportivos que eran compartidos por todos los practicantes. Ninguno de los preparadores que en aquel momento les entrenaban había realizado un curso de preparador específico ni contaba con formación concreta. Ninguno de aquellos preparadores pedía remuneración económica por su trabajo en el gimnasio, dedicación que llevaban a cabo por afición y de manera altruista en todos los casos. Bajo la perspectiva de los diez púgiles entrevistados, aquellos preparadores supieron orientar la práctica boxística hasta convertirla para ellos en una experiencia formativa que ha sido definitiva en sus trayectorias vitales.

En definitiva, el boxeo les proporcionó una oportunidad de promoción social, que en aquel periodo no habrían conseguido de otra manera. Llevaron a cabo actividades totalmente excepcionales para la mayor parte de los jóvenes españoles como viajar o conocer otros entornos sociales y otras poblaciones. Todo ello, con la orientación positiva que recibieron por parte de sus entrenadores, en los diez casos afirmaron que les permitió poder encontrar su identidad personal, superar su autoestima, reconocer su propio valor y adquirir su propia forma de orientar ordenadamente sus conductas vitales. En la actualidad reconocen que el boxeo fue clave en su formación personal y en la orientación positiva de toda su trayectoria laboral y vital.

\section{Referencias}

ABC. (28 de enero de 1968). Asamblea de Federaciones. $A B C$. Recuperado de: http://hemeroteca.abc.es/nav/Navigate.exe/hemeroteca/madrid/abc/1968/01/28/097.ht $\mathrm{ml}$ [2017, 15 de abril]

ABC (23 de enero de 1970). Asamblea de la Asociación de Boxeo "Amateur". ABC. Recuperado de:

http://hemeroteca.abc.es/nav/Navigate.exe/hemeroteca/madrid/abc/1970/01/23/066.ht $\mathrm{ml}[2018,19$ de marzo]

Amorós, A. (1972). Roberto Duque inauguró la Escuela Nacional de Boxeo, una realidad de la F.E.B. FEBOX-BOXEO. 174, 24.

Alfil (28 de enero de 1968). Boxeo: Asamblea de Federaciones. $A B C$. Recuperado de: http://hemeroteca.abc.es/nav/Navigate.exe/hemeroteca/madrid/abc/1968/01/28/097.ht $\mathrm{ml}$ [2018, 19 de marzo]

Antuña-Suárez, A. (s.f.). Asturias Olímpica: Historia de los olímpicos asturianos. Asturias. Servicios de Publicaciones del Principado de Asturias.

Aróstegui-Sánchez, J. (2004). La historia vivida. Sobre la historia del presente. Madrid: Alianza Editorial

Barbero, A. (1989). Historia del Boxeo Aficionado en España. Madrid: Federación Española de Boxeo.

Bédarida, F. (1998). Definición, método y práctica de la Historia del Tiempo Presente. Cuadernos de Historia contemporánea (20) 19-27. Recuperado de:

http://revistas.ucm.es/index.php/CHCO/article/viewFile/CHCO9898110019A/7004 [2017, 23 de abril]

Best, J.M., (1982). Cómo investigar en educación. Madrid: Ediciones Morata.

Biescas, J. A. y Tuñón, M. (1980) España bajo la dictadura franquista (1939-1975). Madrid: Editorial Labor

Calle-Molina, M.T. y Martínez-Gorroño, M.E. (en prensa) José Enrique Rodríguez Cal: Primer medallista español de boxeo olímpico. José Enrique Rodríguez Cal: First Spanish Medalist of Olympic Boxing. Revista Internacional de Medicina y Ciencias de la Actividad Física y el Deporte. Recuperado de: 
Calle-Molina, M. T. y Martínez-Gorroño, M. E. (2018). Historia y circunstancias sociales, deportivas y económicas del boxeo amateur español entre 1960 y 1980. RICYDE. Revista internacional de ciencias del deporte. 54(14), 344-357. https://doi.org/10.5232/ricyde2018.05405

http://cdeporte.rediris.es/revista/inpress/artjose992.pdf [2018, 14 de enero]

Castelló-Traver, J.E. (1988). Siglo XX 1939-1978. Madrid: Anaya.

Cuervo, J. (14 de septiembre de 2015a) José Enrique Rodríguez Cal. "Dacal II", primera entrega. Diario La Nueva España. Sección: Arquitectura personal 1. Recuperado de: http://www.Ine.es/tags/jose-enrique-rodriguez-cal.html [2017, 2 de febrero]

Cuervo, J. (15 de septiembre de 2015b) José Enrique Rodríguez Cal. "Dacal II", segunda entrega. Diario La Nueva España. Sección: Arquitectura personal 1. Recuperado de: http://www.Ine.es/tags/jose-enrique-rodriguez-cal.html [2017, 2 de febrero]

Del Río, C. (28 de octubre de 2012) El perfil: José Enrique Rodríguez Cal "Dacal II", un gancho de primera. Diario El comercio: La voz de Avilés. Recuperado de:

http://www.elcomercio.es/v/20121028/aviles/gancho-primera-20121028.html [2017, 3 de enero]

Díaz, P. (2008). Deportistas andaluces en los Juegos Olímpicos de la Era Moderna: Atenas 1896 a Turín 2006. Sevilla: Fundación Andalucía Olímpica.

FEBOX-BOXEO (1970a). II Trofeo Costa del Sol en Almería. FEBOX-BOXEO. 158, 14.

FEBOX-BOXEO (1970b). Gran éxito en el Torneo "Roberto Duque". FEBOX-BOXEO. 157, 25

FEBOX-BOXEO (1970c). Éxito de los campeonatos interregionales de la confrontación Cantabria-Rioja-Burgos, esta asume la representación de castilla la Vieja. FEBOXBOXEO. 153, 43.

FEBOX-BOXEO (1972). Asamblea anual de federaciones regionales. FEBOX-BOXEO. 173, p.2.

Hammer, D. y Wildavsky, A. (1990). La entrevista semi-estructurada de final abierto. Aproximación a una guía operativa. Historia y Fuente Oral, (4), 23-61.

Martínez-Gorroño, M. E. y Hernández-Álvarez, J. L. (1999). La fuente oral como recurso didáctico en la enseñanza de la Historia. Pamplona: Asociación Española de Americanistas.

Meyer, R. y Girard, C. (1966). El boxeo. Madrid: Comité Olímpico Español.

Montañés, M. (1970). Santander se impuso a Valladolid, en boxeo aficionado. FEBOXBOXEO. 150, p.21.

Radio Televisión Española [RTVE] (1 de enero de 1971). XIX Campeonato de Europa de Boxeo Amateur [Archivo de Vídeo]. Recuperado de:

http://www.rtve.es/alacarta/videos/imagenes-del-deporte/xix-campeonato-europaboxeo-amateur/2837759/ [2018, 19 de marzo]

Sánchez García, R. (2009). Boxeo y proceso de civilización en la sociedad española. Apunts: educación física y deportes. (2) 5-13. Recuperado de: http://www.revista-apunts.com/es/hemeroteca?article=1306 [2018, 19 de marzo]

Soto-Gamboa, A. (2004). Historia del presente: estado de la cuestión y conceptualización. HAOL (3) 101-116. Recuperado de:

http://dialnet.unirioja.es/descarga/articulo/829443.pdf [2017, 2 de julio]

Tamayo-Fajardo, J.A. (2005). Historia de España en los Juegos Olímpicos de verano de la Era Moderna I: 1940-1984. Sevilla: Wanceulen Editorial Deportiva.

Thompson, P. (1988). La voz del pasado. Historia oral. Edicions Alfons el magnanim. Valencia.

Televisión en Asturias [Meta y Persona] (24 de abril de 2008). José Enrique Rodríguez Val "Dacall II" boxeador [Archivo de vídeo]. Recuperado de: https://www.youtube.com/watch?v=]PTubMpbBKA [2017, 3 de septiembre] 